\title{
Niveles de magnesio en pacientes VIH estadio SIDA, que cursan con tuberculosis, criptococosis, y su relación con la respuesta al tratamiento
}

\author{
GERARDO RONCEROS, LUZ HUAROTO, CARLOS ALVARADO-ORTIZ \\ Instituto de Investigaciones Clínicas-Facultad de Medicina-UNMSM.
}

\begin{abstract}
RESUMEN
OBJETIVOS: Demostrar que los pacientes con virus de inmunodeficiencia humana (VIH) estadio síndrome de inmunodeficiencia adquirida (SIDA), asociada a tuberculosis (TBC) o criptococosis, tienen hipomagnesemia, que produce una inadecuada respuesta al tratamiento. DISEÑO: Estudio prospectivo, longitudinal. MATERIAL Y MÉTODOS: Se estudió 300 personas, divididos en seis grupos de 50 personas cada uno: pacientes VIH positivo, pacientes con tuberculosis, pacientes en estadio SIDA con TBC, pacientes en estadio SIDA con criptococosis, pacientes con enfermedades infecciosas agudas y una población normal (donadores de sangre). RESULTADOS: Los valores promedio de magnesio estuvieron significativamente por debajo de los valores normales en los grupos SIDA+TBC, SIDA+criptococosis y TBC. Se aprecia que existe diferencia estadísticamente significativa en los niveles de magnesio en los grupos VIH, SIDA+TBC, SIDA + criptococosis y TBC. Con respecto a la hipomagnesemia y respuesta al tratamiento, el grupo que menos respondió al tratamiento fue el grupo de pacientes con SIDA+TBC (27\%). CONCLUSIONES: La hipomagnesemia es prevalente en los pacientes con infecciones crónicas, con diferencia significativa de los niveles de magnesio entre los grupos estudiados. El grupo VIH estadio SIDA + TBC es el que cursa con mayor anormalidad en las diferentes variables estudiadas.

Palabras clave: Magnesio; síndrome de inmunodeficiencia adquirida; tuberculosis; criptococosis; deficiencia de magnesio.
\end{abstract}

\section{MAGNESIUM LEVELS IN AIDS-ASSOCIATED TUBERCULOSIS OR CRYPTOCOCCOSIS PATIENTS, AND RELATIONSHIP WITH TREATMENT RESPONSE SUMMARY}

OBJECTIVE: To demostrate that human immunodeficiency virus (HIV) patients in acquired immunodeficiency syndrome (AIDS) phase associated with tuberculosis (TB) or cryptococcosis have hypomagnesemia-related inadequate treatment response. DESIGN: Prospective and longitudinal study. MATERIALS AND METHODS: We studied 300 subjects, divided in six groups of 50 patients each: positive HIV patients, TB patients, AIDS patients with TB, AIDS patients with cryptococcosis, no-AIDS patients with acute infections and a normal group (blood donors). RESULTS: Average magnesium values were below normal in the groups AIDS/TB, AIDS/cryptococcosis and TB. There were important statistical differences in magnesium levels in the groups HIV, AIDS/TB, AIDS/ cryptococcosis and TB. With respect to hypomagnesemia, the group with less response to treatment was the AIDS+TB (27\%). CONCLUSIONS: Hypomagnesemia is prevalent in chronic infectious diseases with important differences in magnesium levels among the four groups studied and AIDS/TB group showing more abnormalities.

Key words: Magnesium; acquired immunodeficiency syndrome; tuberculosis; cryptococcosis; magnesium deficiency.

Correspondencia:

Dr. Gerardo Ronceros Medrano

Las Cucardas 127

Lima 32, Perú

E-mail:investcl@terra.com.pe 


\section{INTRODUCCIÓN}

Nuestro país ha ingresado al nuevo milenio con problemas de salud muy serios, propios de la transición epidemiológica en que se encuentra, es decir, un incremento de las enfermedades crónico-degenerativas, pero sin una disminución importante de las patologías infecto-contagiosas $\left({ }^{1}\right)$. Se añade a esto un grave problema de desnutrición, que afecta casi a $60 \%$ de la población $\left({ }^{2}\right)$.

Numerosos estudios, entre ellos los de Bianco, han demostrado la estrecha relación que existe entre las diferentes infecciones, sobre todo las crónicas, y el grado de desnutrición, lo cual incrementa el riesgo de morbilidad y mortali$\operatorname{dad}\left({ }^{3}\right)$.

Por otro lado, algunas enfermedades infecciosas, sobretodo las crónicas -como la tuberculosis (TBC) y actualmente la infección por virus de inmunodeficencia humana (VIH)-, tienen dentro de sus complicaciones a la desnutrición, lo que establece un círculo vicioso entre enfermedad, desnutrición, alteración del sistema inmune $y$ el aumento de infecciones oportunistas $\left({ }^{4-7,8}\right)$.

Se desprende entonces que, sin una adecuada nutrición hay mayor riesgo que se desarrollen enfermedades infecciosas, tanto agudas como crónicas, existiendo la posibilidad de que además no haya una adecuada respuesta al tratamiento y, por consiguiente, prolongar el periodo de recuperación del paciente. Ello influye directamente en el aspecto económico, tanto de los pacientes como de las instituciones que los albergan, que, por ejemplo, en el caso de la tuberculosis es aproximadamente 50 millones de dólares $\left({ }^{9}\right)$.

Dentro de este contexto, se ha observado además una estrecha relación entre el agravamiento de la enfermedad o su inadecuada respuesta al tratamiento y los niveles deficitarios de algunos oligoelementos, especialmente, el magnesio $\left({ }^{5,6}\right)$. Existen diversas publicaciones en las cuales se afirma que la hipomagnesemia estaría directamente involucrada con una serie de enfermedades crónicas $\left({ }^{10,11}\right)$ y que ésta se produciría por un déficit en las cantidades de magnesio que existen en los alimentos.

El magnesio es el segundo catión intracelular más abundante del cuerpo humano, siendo esencial en gran número de procesos enzimáticos y metabólicos.

Se ha apreciado que la disminución de magnesio ocurre principalmente en pacientes hospitalizados, en aquellos que están recibiendo diuréticos que inhiben la reabsorción de sodio en el asa de Henle, en los pacientes que reciben aminoglicósidos, alcohólicos crónicos, pacientes con diarreas crónicas, con reducción de la ingesta, aporte de fluidos intravenosos sin magnesio.

Por otra parte, es importante señalar que la incidencia de algunas enfermedades se ha incrementado sostenidamente en el transcurso de los últimos años. Las enfermedades, como el VIH, la TBC y la criptococosis, son actualmente serios problemas de salud que se agravan aun más por la íntima relación que existe entre ellas.

La infección por VIH se inicia como un cuadro agudo con características de tipo gripal. Esta fase puede pasar inadvertida y la relación con la infección VIH, en muchos casos, no está clara. A la fase aguda le sigue típicamente un estado de portador asintomático, que progresa como promedio dentro de los diez años de la seroconversión hacia el síndrome de inmunodeficiencia adquirida (SIDA) clínico, en $50 \%$ de los individuos infectados $\left({ }^{12}\right)$.

En nuestro país, las principales enfermedades oportunistas relacionadas con el estadio SIDA son la TBC y la criptococosis $\left({ }^{13}\right)$.

La TBC es la enfermedad infectocontagiosa más prevalente en el ámbito mundial, invade a los seres humanos sin distingo de edad, sexo o situación económica. Mueren más enfermos por tuberculosis que por cualquier otra enfermedad; por eso es denominada la primera amenaza de salud pública de la presente década. Desde 1993, la Organización Mundial de la Salud (OMS) ha declarado la TBC como emergencia mundial. 
Por esta razón, la TBC es el prototipo de las infecciones que requieren la respuesta inmune celular para su control. A pesar que la infección está asociada con una rica respuesta de anticuerpos, no se ha demostrado que éstos desempeñen algún papel en los mecanismos de defensa del huésped $\left({ }^{14}\right)$.

La criptococosis es una micosis sistémica producida por un hongo levaduriforme encapsulado denominado Cryptococcus neoformans, descubierto hace aproximadamente cien años. Es una enfermedad de distribución universal, que adquiere protagonismo con la aparición de la epidemia de VIH, en la que existe una diseminación muy rápida al sistema nervioso central (SNC), a consecuencia de una fase primaria pulmonar $\left({ }^{15}\right)$. Antes de la aparición de la epidemia del VIH, era un padecimiento infrecuente y afectaba a pacientes con alguna enfermedad de base que producía una depresión de la inmunidad celular. En la actualidad, es la micosis sistémica más frecuente. Entre 80 y $90 \%$ de todos los casos de criptococosis se producen en estos pacientes. Además, es la principal causa de las infecciones fatales, con una frecuencia que varía entre $2 \mathrm{y}$ $10 \%$ en Europa Occidental y en Norteamérica, hasta alcanzar un nivel de $15 \%$ en ciertas regiones del África $\left({ }^{16}\right)$.

El diagnóstico de la criptococosis se basa en la detección microscópica de la levadura capsulada, en el aislamiento de los Cryptococcus neoformans en cultivo y en la detección de los antígenos capsulares en líquidos biológicos $\left({ }^{17}\right)$.

Estudios preliminares han establecido que pacientes VIH con tuberculosis o criptococosis mostraban una inadecuada respuesta al tratamiento específico cuando presentaban hipomagnesemia, lo cual prolongaba el tiempo de hospitalización y retardaba la mejoría clínica.

El presente trabajo trata de demostrar que la hipomagnesemia de diferente etiología está presente en los pacientes VIH estadio SIDA y, por esta razón, las enfermedades asociadas, como son la tuberculosis y la criptococosis, no responden adecuadamente al tratamiento.

\section{MATERIAL Y MÉTODOS}

El presente es un estudio prospectivo, longitudinal, que se realizó en los pacientes mayores de 18 años de ambos sexos ingresados a los diferentes servicios de infectología de los diferentes hospitales de Lima entre los meses de julio a diciembre del año 2000 , con los siguientes diagnósticos (50 pacientes por grupo):

- Pacientes VIH positivo, con prueba de ELISA para VIH positivo y Western Blot positivo.

- Pacientes con tuberculosis, con frotis positivo para bacilo de Koch (BK).

- Pacientes en estadio SIDA (con dosaje de CD4 menor de 200) con TBC.

- Pacientes en estadio SIDA con criptococosis, con examen de tinta china positivo en el líquido cefalorraquídeo y/o cultivo positivo y/o prueba de látex positiva.

- Pacientes con enfermedades infecciosas agudas respiratorias, salmonelosis e infecciones urinarias, todas con cultivo positivo.

- Una población normal, donadores voluntarios de sangre.

Para la comparación de los diferentes grupos de estudio se utilizó el análisis de varianza (ANOVA) y el método de mínima diferencia significativa (LSD) o método de TAMHANE; adicionalmente se utilizó gráfico de cajas. Todas las evaluaciones fueron realizados a un nivel de significancia del $95 \%$.

A los pacientes se les dosó los niveles de magnesio utilizando como principio la XYLIDYLE (Magon) por el método colorimétrico.

El diagnóstico de VIH positivo se realizó por la técnica de ELISA y el de criptococosis por la técnica de aglutinación, que permite detectar los polisácaridos de la cápsula del criptococo.

En el caso de los pacientes con diagnóstico de tuberculosis, se usó la baciloscopia como téc- 
nica estandarizada (Ziehl Nielsen o tinción ácido alcohol resistente).

La muestra se tomó por punción venosa, en tubos al vacío y se centrifugó a 5000 r.p.m., evitando la hemólisis; cuando esto ocurrió, la muestra fue desechada.

\section{RESULTADOS}

Los valores promedio de magnesio estuvieron significativamente por debajo del valor normal en los grupos de SIDA+TBC, SIDA + criptococosis y TBC, con cifras promedio de 1,6, 1,7 y 1,7 mg/dL, respectivamente. El nivel individual de magnesio más bajo se encontró en el grupo de pacientes con SIDA + TBC y fue de $1,0 \mathrm{mg} / \mathrm{dL}$. El nivel individual máximo de magnesio se apreció en dos pacientes, uno en el grupo de pacientes normales y otro en aquellos con infección aguda, y fue de $3,5 \mathrm{mg} / \mathrm{dL}$.

Como se observa en la Figura 1, los niveles promedio de magnesio por grupo de pacientes estudiados fue el siguiente: pacientes HIV positivos $1,9 \mathrm{mg} / \mathrm{dL}$, pacientes con infecciones agudas $2,5 \mathrm{mg} / \mathrm{dL}$, sujetos normales $2,5 \mathrm{mg} / \mathrm{dL}$,

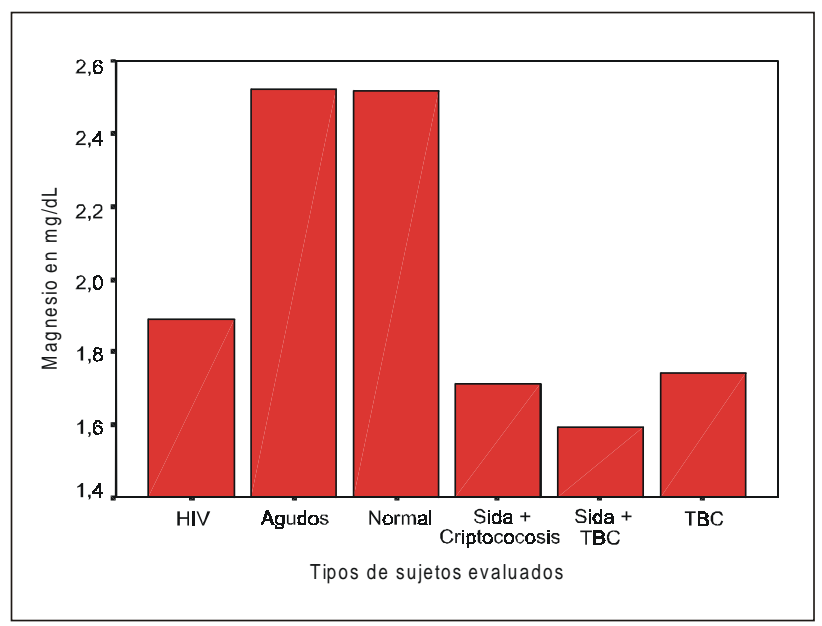

Figura 1.- Nivel sérico de magnesio (mg/dL) por tipo de sujetos.
SIDA + criptococosis $1,7 \mathrm{mg} / \mathrm{dL}$, SIDA + TBC $1,6 \mathrm{mg} / \mathrm{dL}$ y $\mathrm{TBC} 1,7 \mathrm{mg} / \mathrm{dL}$.

En la Tabla 1, Tabla de análisis de múltiples comparaciones de Tamhane, comparamos los niveles de magnesio de cada uno de los grupos con el resto, apreciándose que no existió diferencia estadísticamente significativa entre el grupo de sujetos normales y los pacientes portadores de infecciones agudas, lo que sí se llegó a observar cuando establecimos la comparación con los otros grupos VIH, SIDA+TBC, SIDA + criptococosis y TBC.

El índice de masa corporal (IMC) refleja la relación que existe entre el peso y la talla y es un indicador del estado nutricional de las personas. En este trabajo, el único grupo que tenía un promedio de IMC por debajo del valor normal era el SIDA+TBC, con un valor de 18,1. En el resto de los grupos, los valores promedio eran normales, aquellos de los pacientes con SIDA + criptococosis y TBC con valores de 19,3 y 20,1 , respectivamente, fueron sustantivamente menores que los de VIH, normales y de infección aguda.

Los promedios de índice de masa corporal se aprecia en el Figura 2 y fueron los siguientes:

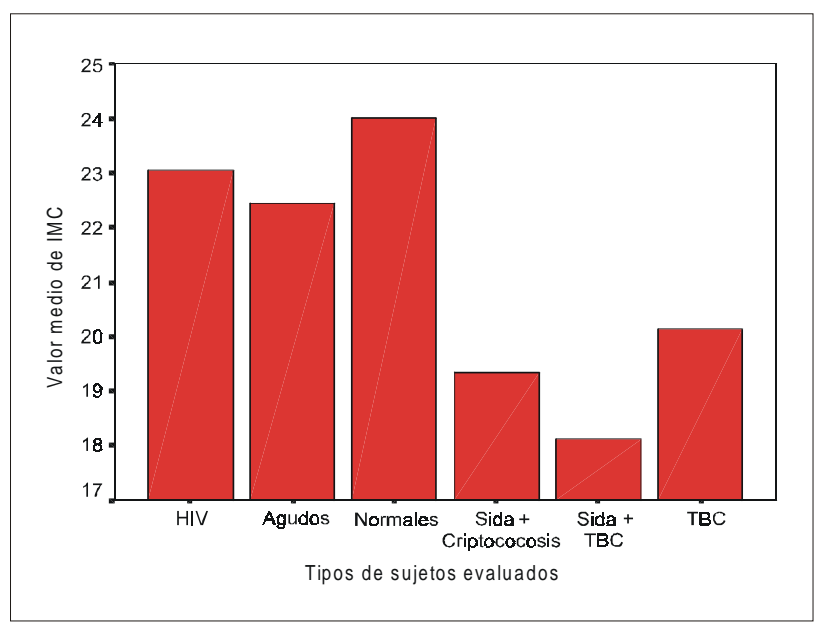

Figura 2.- Índice de masa corporal (IMC) por tipo de sujetos. 
Tabla 1. Comparaciones múltiples: Tamhane Variable dependiente: $\mathrm{Mg}$

\begin{tabular}{|c|c|c|c|c|c|c|}
\hline \multicolumn{2}{|c|}{ Magnesio } & \multirow{2}{*}{$\begin{array}{c}\text { Diferencia media } \\
(\mathrm{I}-\mathrm{J})\end{array}$} & \multirow{2}{*}{$\begin{array}{l}\text { Error } \\
\text { estándar }\end{array}$} & \multirow{2}{*}{ Significación } & \multicolumn{2}{|c|}{ Intervalo confiabilidad $95 \%$} \\
\hline (I) Grupo & (J) Grupo & & & & Nivel bajo & Nivel alto \\
\hline \multirow[t]{5}{*}{ Normal } & VIH & 0,6290 & $6,61 \mathrm{E}-02$ & 0,000 & 0,4357 & 0,8223 \\
\hline & Agudo & $-4,40 \mathrm{E}-03$ & $6,61 \mathrm{E}-02$ & 1,000 & $-0,2268$ & 0,2180 \\
\hline & Sida-Cript & 0,8056 & $6,61 E-02$ & 0,000 & 0,5915 & 1,0197 \\
\hline & Sida-TBC & 0,9264 & $6,61 \mathrm{E}-02$ & 0,000 & 0,7234 & 1,1294 \\
\hline & TBC & 0,7756 & $6,61 E-02$ & 0,000 & 0,5928 & 0,9584 \\
\hline \multirow[t]{5}{*}{ VIH } & Normal & $-0,6290$ & $6,61 \mathrm{E}-02$ & 0,000 & $-0,8223$ & $-0,4357$ \\
\hline & Agudo & $-0,6334$ & $6,61 E-02$ & 0,000 & $-0,8395$ & $-0,4273$ \\
\hline & Sida-Cript & 0,1766 & $6,61 E-02$ & 0,118 & $-2,03 \mathrm{E}-02$ & 0,3735 \\
\hline & Sida-TBC & 0,2974 & $6,61 \mathrm{E}-02$ & 0,000 & 0,1128 & 0,4820 \\
\hline & $\mathrm{TBC}$ & 0,1476 & $6,61 E-02$ & 0,109 & $-1,50 \mathrm{E}-02$ & 0,3082 \\
\hline \multirow[t]{5}{*}{ Agudo } & Normal & $4,40 \mathrm{E}-03$ & $6,61 E-02$ & 1,000 & $-0,218$ & 0,2268 \\
\hline & VIH & 0,6334 & $6,61 \mathrm{E}-02$ & 0,000 & $4,27 \mathrm{E}-01$ & 0,8395 \\
\hline & Sida-Cript & 0,8100 & $6,61 \mathrm{E}-02$ & 0,000 & 0,5845 & 1,0355 \\
\hline & Sida-TBC & 0,9308 & $6,61 \mathrm{E}-02$ & 0,000 & $7,16 \mathrm{E}-01$ & 1,1459 \\
\hline & TBC & 0,7800 & $6,61 E-02$ & 0,000 & 0,5835 & 0,9765 \\
\hline \multirow[t]{5}{*}{ Sida-Cript } & Normal & $-0,8056$ & $6,61 \mathrm{E}-02$ & 0,000 & $-1,02 \mathrm{E}+00$ & $-0,5915$ \\
\hline & VIH & $-0,1766$ & $6,61 E-02$ & 0,118 & $-0,3735$ & $2,03 \mathrm{E}-02$ \\
\hline & Agudo & $-0,8100$ & $6,61 \mathrm{E}-02$ & 0,000 & $-1,04 \mathrm{E}+00$ & $-0,5845$ \\
\hline & Sida-TBC & 0,1208 & $6,61 \mathrm{E}-02$ & 0,723 & $-8,56 \mathrm{E}-02$ & 0,3272 \\
\hline & TBC & $-3,00 \mathrm{E}-02$ & $6,61 E-02$ & 1,000 & $-2,17 \mathrm{E}-01$ & 0,1567 \\
\hline \multirow[t]{5}{*}{ Sida-TBC } & Normal & $-0,9264$ & $6,61 \mathrm{E}-02$ & 0,000 & $-1,13 \mathrm{E}+00$ & $-0,7234$ \\
\hline & $\mathrm{VIH}$ & $-0,2974$ & $6,61 \mathrm{E}-02$ & 0,000 & $-4,82 \mathrm{E}-01$ & $-0,1128$ \\
\hline & Agudo & $-0,9308$ & $6,61 E-02$ & 0,000 & $-1,15 \mathrm{E}+00$ & $-0,7157$ \\
\hline & Sida-Cript & $-0,1208$ & $6,61 E-02$ & 0,723 & $-3,27 \mathrm{E}-01$ & $8,56 \mathrm{E}-02$ \\
\hline & ТВC & $-0,1508$ & $6,61 \mathrm{E}-02$ & 0,146 & $-3,24 \mathrm{E}-01$ & $2,26 \mathrm{E}-02$ \\
\hline \multirow[t]{5}{*}{ TBC } & Normal & $-0,7756$ & $6,61 E-02$ & 0,000 & $-9,58 \mathrm{E}-01$ & $-0,5928$ \\
\hline & VIH & $-0,1466$ & $6,61 \mathrm{E}-02$ & 0,109 & $-3,08 \mathrm{E}-01$ & 1,50E-02 \\
\hline & Agudo & $-0,7800$ & $6,61 \mathrm{E}-02$ & 0,000 & $-9,77 E-01$ & $-5,84 \mathrm{E}-01$ \\
\hline & Sida-Cript & $3,00 \mathrm{E}-02$ & $6,61 E-02$ & 1,000 & $-1,57 \mathrm{E}-01$ & $2,17 \mathrm{E}-01$ \\
\hline & Sida-TBC & 0,1508 & $6,61 \mathrm{E}-02$ & 0,146 & $-2,26 \mathrm{E}-02$ & $3,24 \mathrm{E}-01$ \\
\hline
\end{tabular}

pacientes VIH positivos 23 , pacientes con infecciones agudas 22,4 , sujetos normales 24 , SIDA + criptococosis 19,3, SIDA + TBC 18,1 y TBC 20,1 .

En la Tabla 2, se observa que según el análisis de múltiples comparaciones, no existió diferencia estadísticamente significativa entre los IMC de los sujetos normales con los de VIH y los pacientes portadores de enfermedades agudas; por el contrario, se apreció que existía diferencia estadísticamente significativa con el resto de los grupos SIDA+TBC, SIDA+ criptococosis y TBC.
En la Figura 3, evaluamos la respuesta al tratamiento al comparar cuatro grupos de pacientes, aquellos que tuvieron una infección aguda de diferente etiología y en quienes se utilizó antibioticoterapia específica contra el germen detectado.

Este grupo presentó una respuesta adecuada de $100 \%$ (50). Los pacientes tuberculosos tuvieron una respuesta al tratamiento antituberculoso estándar en $76 \%$ (39) de los casos, el grupo de SIDA + criptococosis $68 \%$ (34) de respuesta y el grupo que respondió menos al tratamiento fue el de SIDA+TBC, en $46 \%$ de los casos (23). 
Tabla 2. Comparaciones múltiples: Tamhane

Variable dependiente: Índice de masa corporal

\begin{tabular}{|c|c|c|c|c|c|c|}
\hline \multicolumn{2}{|c|}{ IMC } & \multirow{2}{*}{$\begin{array}{l}\text { Diferencia media } \\
\text { (I-J) }\end{array}$} & \multirow{2}{*}{$\begin{array}{l}\text { Error } \\
\text { estándar }\end{array}$} & \multirow{2}{*}{ Significación } & \multicolumn{2}{|c|}{ Intervalo confiabilidad $95 \%$} \\
\hline (I) Grupo & (J) Grupo & & & & Nivel bajo & Nivel alto \\
\hline \multirow[t]{5}{*}{ Normal } & VIH & 0,9460 & $7,20 \mathrm{E}-01$ & 0,990 & $-1,5759$ & 3.4679 \\
\hline & Agudo & $1,56 \mathrm{E}+00$ & $7,20 \mathrm{E}-01$ & 0,627 & $-0,9391$ & 4.0591 \\
\hline & Sida-Cript & 4,6640 & $7,20 \mathrm{E}-01$ & 0,000 & 2,2104 & 7.1176 \\
\hline & Sida-TBC & 5,8760 & $7,20 \mathrm{E}-01$ & 0,000 & 3,4562 & 8.2958 \\
\hline & TBC & 3,8480 & $7,20 \mathrm{E}-01$ & 0,000 & 1,4412 & 6.2548 \\
\hline \multirow[t]{5}{*}{ VIH } & Normal & $-0,9460$ & $7,20 \mathrm{E}-01$ & 0,990 & $-3,4679$ & 1.5759 \\
\hline & Agudo & 0,6140 & $7,20 \mathrm{E}-01$ & 0,999 & $-1,5073$ & 2.7353 \\
\hline & Sida-Cript & 3,7180 & $7,20 \mathrm{E}-01$ & 0,000 & $1,65 \mathrm{E}+00$ & 5.7836 \\
\hline & Sida-TBC & 4,9300 & $7,20 \mathrm{E}-01$ & 0,000 & 2,906 & 6.9540 \\
\hline & $\mathrm{TBC}$ & 2,9020 & $7,20 \mathrm{E}-01$ & 0,001 & $8,94 \mathrm{E}-01$ & 4.9099 \\
\hline \multirow[t]{5}{*}{ Agudo } & Normal & $-1,56 \mathrm{E}+00$ & $7,20 \mathrm{E}-01$ & 0,627 & $-4,0591$ & 0.9391 \\
\hline & VIH & $-0,6140$ & $7,20 \mathrm{E}-01$ & 0,999 & $-2,74 \mathrm{E}+00$ & 1.5073 \\
\hline & Sida-Cript & 3,1040 & $7,20 \mathrm{E}-01$ & 0,000 & 1,0674 & 5.1406 \\
\hline & Sida-TBC & 4,3160 & $7,20 \mathrm{E}-01$ & 0,000 & $2,32 \mathrm{E}+00$ & 6.3103 \\
\hline & TBC & 2,2880 & $7,20 \mathrm{E}-01$ & 0,012 & 0,3101 & 4.2659 \\
\hline \multirow[t]{5}{*}{ Sida-Cript } & Normal & $-4,6640$ & $7,20 \mathrm{E}-01$ & 0,000 & $-7,12 \mathrm{E}+00$ & -2.2104 \\
\hline & VIH & $-3,7180$ & $7,20 \mathrm{E}-01$ & 0,000 & $-5,7836$ & $-1.65 E+00$ \\
\hline & Agudo & $-3,1040$ & $7,20 \mathrm{E}-01$ & 0,000 & $-5,14 \mathrm{E}+00$ & -1.0674 \\
\hline & Sida-TBC & 1,2120 & $7,20 \mathrm{E}-01$ & 0,623 & $-7,22 \mathrm{E}-01$ & 3.1461 \\
\hline & TBC & $-8,16 \mathrm{E}-01$ & $7,20 \mathrm{E}-01$ & 0,968 & $-2,73 E+00$ & 1.1012 \\
\hline \multirow[t]{5}{*}{ Sida-TBC } & Normal & $-5,8760$ & $7,20 \mathrm{E}-01$ & 0,000 & $-8,30 \mathrm{E}+00$ & -3.4562 \\
\hline & VIH & $-4,9300$ & $7,20 \mathrm{E}-01$ & 0,000 & $-6,95 E+00$ & -2.906 \\
\hline & Agudo & $-4,3160$ & $7,20 \mathrm{E}-01$ & 0,000 & $-6,31 E+00$ & -2.3217 \\
\hline & Sida-Cript & $-1,2120$ & $7,20 \mathrm{E}-01$ & 0,623 & $-3,15 E+00$ & $7.22 \mathrm{E}-01$ \\
\hline & TBC & $-2,0280$ & $7,20 \mathrm{E}-01$ & 0,023 & $-3,90 E+00$ & $-1.57 \mathrm{E}-01$ \\
\hline \multirow[t]{5}{*}{ TBC } & Normal & $-3,8480$ & $7,20 \mathrm{E}-01$ & 0,000 & $-6,25 E+00$ & -1.4412 \\
\hline & VIH & $-2,9020$ & $7,20 \mathrm{E}-01$ & 0,001 & $-4,91 \mathrm{E}+00$ & $-8.94 \mathrm{E}-01$ \\
\hline & Agudo & $-2,2880$ & $7,20 \mathrm{E}-01$ & 0,012 & $-4,27 \mathrm{E}+00$ & $-3.10 \mathrm{E}-01$ \\
\hline & Sida-Cript & $8,16 \mathrm{E}-01$ & $7,20 \mathrm{E}-01$ & 0,968 & $-1,10 \mathrm{E}+00$ & $2.73 E+00$ \\
\hline & Sida-TBC & 2,0280 & $7,20 \mathrm{E}-01$ & 0,023 & $1,57 \mathrm{E}-01$ & $3.90 \mathrm{E}+00$ \\
\hline
\end{tabular}

En la Tabla 3, se aprecia la relación que existió entre la respuesta al tratamiento y los niveles de magnesio, en cuatro grupos. Los que tuvieron enfermedad infecciosa aguda presentaron hipomagnesemia en $2 \%$ (1), con una respuesta adecuada al tratamiento de $100 \%$. En el grupo de TBC, $60 \%$ (30) de los pacientes presentó hipomagnesemia y de éstos 63,4\%(19) respondió adecuadamente al tratamiento. $\mathrm{Y}$, de los normomagnesémicos, $100 \%$ (26) respondió al tratamiento. En el grupo SIDA + criptococosis, $62 \%(31)$ de los pacientes presentó hipomagnesemia y $48,4 \%$ (15) tuvo buena respuesta al tratamiento; $38 \%$ (19) fueron normomagnesémicos, con respuesta adecuada al tratamiento en $94,7 \%$ (18). Finalmente, en el grupo SIDA + TBC, 74\% (37) de los pacientes presentó hipomagnesemia, con una buena respuesta al tratamiento en $27 \%$ (10). Y los normomagnesémicos fueron $26 \%$ (13), de los cuales $84,6 \%$ tuvo una adecuada respuesta al tratamiento (11).

\section{DISCUSIÓN}

Desde 1921, con los estudios de Krieger realizados en pacientes tuberculosos y después con 


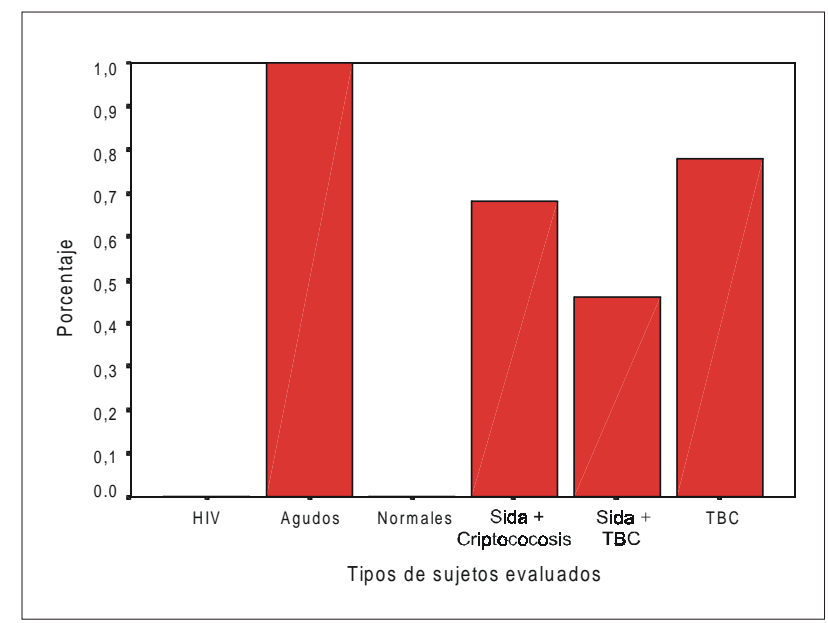

Figura 3.- Respuesta al tratamiento por tipo de sujetos.

los realizados tanto por Brozek como Winick en los prisioneros judíos de los ghetos de Varsovia, Holanda y Leningrado, se demostró que la desnutrición -por ella misma- aumenta la morbilidad y la mortalidad. Se conoce que la pérdida de más de $40 \%$ de peso corporal es fatal por sí sola, considerándose la principal causa de muerte sin evidencia de enfermedad infecciosa o tumoral $\left({ }^{4}\right)$.
En nuestro país, esta situación se agrava por la presencia no sólo de la desnutrición y de las infecciones crónicas, como la tuberculosis, sino también por la aparición en los últimos 20 años de la infección del virus de la inmunodeficiencia humana (VIH). La confluencia de estas patologías condiciona una serie de situaciones, como la disminución de macronutrientes y micronutrientes, entre ellos principalmente el magnesio y el zinc. La disminución de los niveles de zinc está directamente relacionada con la alteración de la respuesta inmune en los pacientes, lo cual influye en la respuesta al tratamiento.

No hay estudios en nuestro medio que describan la relación que existe entre los niveles de magnesio, las infecciones crónicas y su relación con la respuesta al tratamiento.

Los niveles de magnesio no se modificaron en los sujetos control ni en aquellos con infecciones agudas (salmonelosis, enfermedades respiratorias agudas, infecciones urinarias) que ingresaron al estudio. Este comportamiento parece estar en relación al tiempo que dura el proceso, el que por ser muy corto no permite que el magnesio sufra la disminución que se aprecia en los otros grupos de pacientes, sobretodo en los pacientes con SIDA+TBC. Al parecer hay

Tabla 3. Magnesio y respuesta al tratamiento.

\begin{tabular}{cccccccc}
\hline \multicolumn{4}{c}{ Agudos } & \multicolumn{3}{c}{ TBC } \\
\hline \multicolumn{2}{c}{ HipoMg 1 $(2 \%)$} & \multicolumn{2}{c}{ NormoMg $49(98 \%)$} & HIpoMg $30(60 \%)$ & NormoMg 26 (40\%) \\
\hline BR & MR & BR & MR & BR & MR & BR & MR \\
\hline $1(100 \%)$ & $0(0 \%)$ & $49(100 \%)$ & $0(0 \%)$ & $19(63,4 \%)$ & $11(36,6 \%)$ & $26(100 \%)$ & $0(0 \%)$ \\
\hline
\end{tabular}

SIDA + Criptococosis

SIDA + TBC

\begin{tabular}{cccccccc}
\hline \multicolumn{2}{c}{ HipoMg $31(62 \%)$} & \multicolumn{2}{c}{ NormoMg $19(38 \%)$} & \multicolumn{2}{c}{ HipoMg $37(74 \%)$} & \multicolumn{2}{c}{ NormoMg $13(26 \%)$} \\
\hline BR & MR & BR & MR & BR & MR & BR & MR \\
\hline $15(48,38 \%)$ & $16(51,62 \%)$ & $18(94,73 \%)$ & $1(5,27 \%)$ & $10(27,03 \%)$ & $27(72,97 \%)$ & $11(84,6 \%)$ & $2(15,4 \%)$ \\
\hline
\end{tabular}

HipoMg = Hipomagnesemia

NormoMg = Normomagnesemia

$B R=$ Buena respuesta

$M R=$ Mala respuesta 
una relación muy estrecha entre la cronicidad del proceso infeccioso y la disminución de los niveles de magnesio, debido probablemente a la disminución de la ingesta, a las pérdidas incrementadas a través del tracto gastrointestinal y a la inadecuada reposición de magnesio en los pacientes que necesitan tratamiento con fluidos por vía parenteral.

Es importante señalar que algunos de nuestros pacientes tenían niveles de magnesio por encima de los valores normales, inclusive algunos con manifiesta hipermagnesemia, sin que aparentemente tuvieran sintomatología clínica propia de la misma, como parálisis neuromuscular periférica, hipotonía, apnea o trastornos de la coagulación. Es probable que la sintomatología haya existido, pero por el poco conocimiento de la misma se haya confundido con el cuadro clínico de fondo.

Por otra parte, los valores normales con lo que estamos trabajando se refieren a sujetos europeos o norteamericanos, que tienen una realidad nutricional y patológica diferente. Por eso sería conveniente establecer en nuestra población los valores normales con respecto al magnesio. En nuestro trabajo, al evaluar los resultados del grupo de pacientes normales con respecto a sus niveles de magnesio, apreciamos como límite mínimo $1,8 \mathrm{~mL} / \mathrm{dL}$ y como límite máximo 3,5 $\mathrm{mL} / \mathrm{dL}$ con una media de $2,5 \mathrm{~mL} / \mathrm{dL}$.

El grupo de pacientes con infección por VIH tiene niveles de magnesio en el limite inferior normal, comportándose de una manera diferente con el grupo de normales y pacientes con infecciones agudas, a pesar que es un paciente que aún no tiene sintomatología propia de la enfermedad; probablemente, al pasar estos pacientes al estadio SIDA, la hipomagnesemia se establece rápidamente. De esta forma podríamos establecer la relación entre infección crónica e hipomagnesemia, aunque no podemos aun definir la relación causa efecto.

En cuanto al índice de masa corporal, los valores obtenidos estarían reflejando que es en las enfermedades crónicas en donde se produce el mayor grado de desnutrición, lo que se relacionaría con la menor ingesta de alimentos y probablemente con la mayor pérdida a través del tracto gastrointestinal. A todo esto debemos agregar que en nuestro país existe alto índice de desnutrición, que acompaña a la crisis económica, al alto grado de desempleo y subempleo existente, que no permiten una adecuada alimentación. También es importante señalar que el nivel educativo de la población impide que se aproveche apropiadamente los recursos naturales existentes, lo que agrava el cuadro anteriormente descrito.

Cuando analizamos la respuesta al tratamiento, apreciamos que la relación entre el VIH-estadio SIDA y TBC es la que tiene mayor índice de fracasos. Es conocido que los pacientes con infección VIH que adquieren la tuberculosis o hacen una recidiva y que no responden al tratamiento, es por que el Mycobacterium tuberculosis causante de la infección es una cepa que se ha hecho multidrogorresistente, al haber estado expuesta ya al tratamiento estándar. Este hecho produce una gran mortalidad en este grupo de pacientes y un problema epidemiológico, con respecto al contagio, que resulta muy difícil de solucionar en el momento actual.

La relación entre la hipomagnesemia y la respuesta al tratamiento es la parte más importante del presente trabajo, apreciándose que en los grupos hipomagnesémicos la respuesta al tratamiento es menor, sobre todo en el grupo VIH estadio SIDA + TBC, en donde $74 \%$ de los pacientes hipomagnesémicos tuvo una mala respuesta al tratamiento. En el grupo VIH estadio SIDA + criptococosis, $51 \%$ de los hipomagnesémicos tuvo una mala respuesta al tratamiento. No se puede establecer con claridad cuál es la causa de la relación entre los niveles de magnesio y la respuesta al tratamiento, pero es evidente que el magnesio debe cumplir alguna función en el ingreso o en el transporte de los antibióticos, o en los procesos inmunológicos normales.

Es necesario, para demostrar realmente la existencia de una relación directa de la hipo- 
magnesemia y la respuesta al tratamiento, diseñar un estudio en el cual se administre magnesio como parte del tratamiento de la enfermedad infecciosa crónica de fondo.

Se concluye que la hipomagnesemia es prevalente en los pacientes del presente estudio con infecciones crónicas. Existe diferencia entre los niveles de magnesio sérico de los diferentes grupos de pacientes. La relación VIH estadio SIDA + TBC es la que cursa con mayor anormalidad en las diferentes variables estudiadas. Existe diferencia estadísticamente significativa en la respuesta al tratamiento de los grupos estudiados. Creemos conveniente introducir dosaje de magnesio antes de la instalación de la terapia en todos los pacientes con infecciones crónicas. En los casos de hipomagnesemia inicial sería recomendable la reposición de magnesio hasta niveles normales.

\section{BIBLIOGRAFÍA}

1. Documento Lineamientos de Políticas de Salud del Ministerio de Salud. Documento oficial del Ministerio de Salud. 1995-2000.

2. Pajuelo J, Villanueva M,Chávez J. La Desnutrición Crónica, el Sobrepeso y la Obesidad en Niños de Areas Rurales del Perú. An Fac Med1998; 61(3): 201-6.

3. Bianco NE. The immunopathology of systemic anergy in infectious diseases: A reappraisal and new perspectives. Clin Immunol Immmunopathol 1992; 62: 253-7.

4. Nerad JL, Howard SL. Nutrition in the HIV-infected patient undergoing surgery. Samuel E. Wilson, ed. Surgical Problems in the AIDS Patient. Igaku - Shoin. 1994: 20734.
5. Heise W, Nehm K, L'Age M, Averdunk R, Gunther T. Concentrations of magnesium, zinc and copper in serum of patients with acquired immunodeficiency syndrome. J Clin Chem Clin Biochem 1989; 27: 515-7.

6. Fordyce-Baum MK, Mantero-Atienza E, Van Reil F, et al. Nutrition abnormalities in early HIV-1 infection II. Trace elements (abstract). Proceedings of the 5th International Conference on AIDS, June 4-9, 1989, Montreal, Canada.

7. Ysseldyke LL. Nutritional complications and incidence of malnutrition among AIDS patients. J Am Diet Assoc 1991; 91: 217-8.

8. Suárez G y col. Tuberculosis en el Perú 2000, Ministerio de Salud. Dirección General de Salud de las Personas. Programa Nacional de Control de Enfermedades Transmisibles-Control de Tuberculosis.

9. Suárez G. Impacto económico de la tuberculosis en el Perú. Ministerio de Salud-USAID-Vigía. 1999.

10. Patarca R. Universidad de Miami. htp://www.salud.com/ salud/CDA/Article.1,1231,8-33-2389,00.html

11. Vidal NA, Ahmed MA y col. Revista Cubana Aliment Nutr 1998; 12(1): 29-34.

12. Pair JP, Chadwick MD. The biologic and clinical basis of infectious diseases, eds Shulman ST, Pair JP, Sommers HM. Philadelphia: WB Saunders, 1992: 383.

13. Tellería J y col. Enfermedades de Transmisión Sexual. http:/ /webs.demasiado.com/disfunsex/sida.htm

14. Cameron ML, Bartlett JA, Gallis HA, Waskin HA. Manifestations of pulmonary cryptococcosis in patients with acquired immunodeficiency syndrome. RID 1991; 13: 64-7.

15. Dromer F, Mathoulin S, Dupont B, Laporte A. Frech Cryptococcosis Study Group. Epidemiology of cryptococcosis in France: A 9-year survery (1985-1993). Clin Inf Dis 1996; 23: 82-90.

16. Staib F. Criptococcosis in AIDS - Mycological-diagnostic and epidemiological observations. Alds- Forshung (AIFO) 1987: 363-82.

17. Mandell G, Bennet J, Dolin R. Principios y Prácticas de Enfermedades Infecciosas. V Edición. Interamericana vol 2; pag. 2576-83. 